\title{
A Paradox about the Analytic Signal of Laser Beams
}

\author{
B. Lacaze*
}

TeSA 14/16 Port St-Etienne 31000, Toulouse, France

\begin{abstract}
In the signal theory framework the "analytic signal" is a particular polar representation of a signal $f(t)$. It defines an envelope (a modulus) and a phase (an argument) and these parameters are basic in communications. In this paper, we show that amplitude demodulations generally change envelopes and reduced phases. Consequently we show that recent results in laser communications about these parameters are erroneous.
\end{abstract}

Keywords: Analytic signal, laser propagation, envelope.

\section{INTRODUCTION}

Atmospheric turbulence induces limitations of performances in optical communications. Recent papers study fluctuations in laser propagation on distances going to dozens of kilometres [1-3]. To measure influence of atmosphere, the received beam is mixed with a delayed version and frequency downshifted of the transmitted wave. The delay is in the order of magnitude of the propagation time to maintain the beam coherence. A first frequency downshift is obtained from an AOM (acousto-optic modulator) which puts the wave in an intermediary frequency (in the order of few $100 \mathrm{MHz}$ ). This electrical signal holds the information about the laser beam. A second demodulation sends the spectrum in baseband.

The envelope gives insights about the widening of the laser wave. Nevertheless, demodulations introduce changes about envelopes and this drawback seems to be forgotten by researchers. This paper establishes links between envelopes and phases before and after demodulations. Computations are done in the deterministic framework rather than in the random framework because examples are easier to construct.

\section{ENVELOPE AND PHASE}

We give some definitions in the signal theory framework.

1) Let $f(t)$ be a function with real values. The Fourier transform $F(\omega)$ is defined by

$f(t)=\int_{-\infty}^{\infty} F(\omega) e^{i \omega t} d \omega$

The Hilbert transform $\mathcal{H}[f]$ is defined by

$\mathcal{H}[f](t)=\int_{-\infty}^{\infty} \frac{f(u)}{\pi(t-u)} d u$

The analytic signal $\tilde{f}$ of $f$ is defined by $[4,5]$

$\tilde{f}=f+i \mathcal{H}[f]$.

*Address correspondence to this author at the TeSA 14/16 Port St-Etienne 31000, Toulouse, France; Tel: 0561247360; Fax: 0561-2473-73;

E-mail: bernard.lacaze@ esa.prd.fr $\tilde{f}(t)$ is a complex number. If $f_{+}$and $f_{-}$are the components of positive and negative frequency of $f$

$f^{+}(t)=\int_{0}^{\infty} F(\omega) e^{i \omega t} d \omega$
$f^{-}(t)=\int_{-\infty}^{0} F(\omega) e^{i \omega t} d \omega$.

Then we have

$\tilde{f}=2 f_{+}$

An amplitude demodulation $D_{\omega_{0}}^{\varphi}$ of shift $\omega_{0}>0$ is the operation which leads to

$D_{\omega_{0}}^{\varphi}[f](t)=f_{+}(t) e^{-i \omega_{0}(t+\varphi)}+f_{-}(t) e^{i \omega_{0}(t+\varphi)}$

where $\varphi$ is some phase defined by devices and times of propagation. In communications, the signal $f(t)$ is multiplied by $2 \cos \omega_{0}(t+\varphi)$ and filtered in baseband. Roughly speaking, the multiplication by $2 \cos \omega_{0}(t+\varphi)$ changes the frequential components at $\omega$ in two components at $\omega+\omega_{0}$ and $\omega-\omega_{0}$. The first one (resp. the second one) is cancelled by a lowpass filter for $\omega>0$ (resp. $\omega<0)$. The result is $D_{\omega_{0}}^{\varphi}[f]$.

2) The envelope $\mathcal{E}[f]$ and the phase $\mathcal{F}[f]$ of $f$ are defined as the modulus and the argument (modulo $2 \pi$ ) of the analytic signal $\tilde{f}$ :

$$
\mathcal{E}[f]=\sqrt{f^{2}+\mathcal{H}[f]^{2}}, \mathcal{F}[f]=\arg \tilde{f} .
$$

In real conditions $F(\omega)$ (the "spectrum" of $f$ ) cancels outside the neighborhood of some frequencies for instance $\pm \omega_{0}$. In a first step $f$ is shifted in baseband by a demodulation. The result is a new function whose spectrum cancels outside a neighborhood of the frequency 0 . The envelope and the phase of this last function are then computed. It seems commonly admitted that this operation does not change the envelope value.

If $f$ is split in four parts

$f_{-2}(t)=\int_{-\infty}^{-\omega_{0}} F(\omega) e^{i \omega t} d \omega$

2013 Bentham Open 


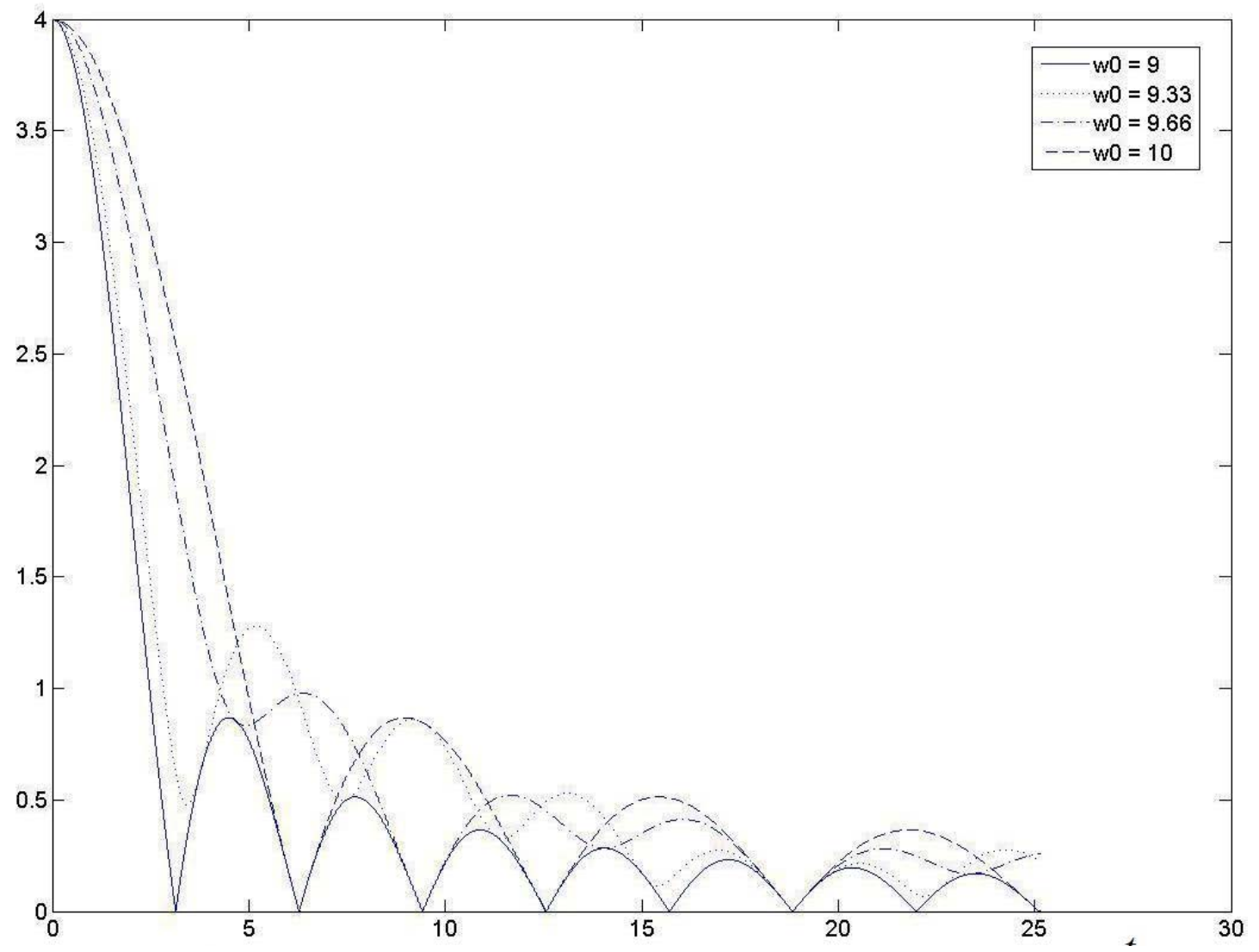

Fig. (1). Envelope for 4 values of the frequency shift.

$$
\begin{aligned}
& f_{-1}(t)=\int_{-\omega_{0}}^{0} F(\omega) e^{i \omega t} d \omega \\
& f_{1}(t)=\int_{0}^{\omega_{0}} F(\omega) e^{i \omega t} d \omega \\
& f_{2}(t)=\int_{\omega_{0}}^{\infty} F(\omega) e^{i \omega t} d \omega
\end{aligned}
$$

then we have, from (5) , (6)

$$
\begin{aligned}
& \widetilde{D}_{\omega_{0}}^{\varphi}[f]=2 f_{-1}(t) e^{i \omega_{0}(t+\varphi)}+2 f_{2}(t) e^{-i \omega_{0}(t+\varphi)} \\
& \tilde{f}(t)=2 f_{1}(t)+2 f_{2}(t)
\end{aligned}
$$

where $\widetilde{D}_{\omega_{0}}^{\varphi}[f]$ is the analytic signal of $D_{\omega_{0}}^{\varphi}$ (the demodulated version of $f$ ). Consequently to (9) we are sure that the demodulation does not change the envelope when $f_{-1}$ and $f_{1}$ are the null function $\left(f_{-1}\right.$ and $f_{1}$ are conjugate functions because $f$ is assumed real). It is the case when the frequency bands of $f$ do not intersect the interval $\left(-\omega_{0}, \omega_{0}\right)$, The property is untrue in other cases.

3) For instance we consider the function

$$
f(t)=\int_{-11}^{-9}+\int_{9}^{11} e^{i \omega t} d \omega=4 \frac{\sin t}{t} \cos 10 t .
$$

When $\omega_{0}=10, \varphi=10$, we have, from (8)

$$
\begin{aligned}
& f_{-2}(t)=\frac{e^{-11 i t}-e^{-10 i t}}{i t} \\
& f_{-1}(t)=\frac{e^{-9 i t}-e^{-10 i t}}{i t} \\
& f_{2}(t)=\frac{e^{11 i t}-e^{10 i t}}{i t}
\end{aligned}
$$

$f_{1}(t)=\frac{e^{10 i t}-e^{9 i t}}{i t}$

And also, from (4), (6), (9)

$D_{10}^{0}[f](t)=\frac{4 \sin t}{t}, \widetilde{D}_{10}^{0}[f](t)=\frac{8 \sin (t / 2)}{t} e^{i t / 2}$,

$\tilde{f}(t)=\frac{4 \sin t}{t} e^{10 i t}$

Clearly the envelopes of $f$ and of its demodulation $D_{10}^{0}[f]$ are different. The first one is equal to $4|\sin t / t|$ and the second one is $8\left|\frac{\sin (t / 2)}{t}\right|$. For other values of $\omega_{0}$ the results are different. When $0<\omega_{0}<9$, the envelope does not change, which is not the case for $9<\omega_{0}<11$. Fig. (1) depicts the envelope for some values of $\omega_{0}$, Computations are straightforward.

4) In this example we have a problem of aliasing, drawback which is well known in sampling theory. In the demodulation process, the frequency bands $(-11,-9)$ and $(9$, 11) are folded on the same interval $(-1,1)$. The band $(-11$, $10)$ goes up to $(-1,0)$ where the band $(9,10)$ goes down. To avoid this drawback, it suffices to measure $\widetilde{D}_{\omega_{0}}^{\varphi}[f]$ for two different values $\varphi_{1}, \varphi_{2}$ of $\varphi$. For instance

$\widetilde{D}_{\omega_{0}}^{\varphi_{1}}[f](t)=2 f_{-1}(t) e^{i \omega_{0}\left(t+\varphi_{1}\right)}+2 f_{2}(t) e^{-i \omega_{0}\left(t+\varphi_{1}\right)}$
$\widetilde{D}_{\omega_{0}}^{\varphi_{2}}[f](t)=2 f_{-1}(t) e^{i \omega_{0}\left(t+\varphi_{2}\right)}+2 f_{2}(t) e^{-i \omega_{0}\left(t+\varphi_{2}\right)}$

which leads to $\left(f_{-1}=f_{1}^{*}\right.$ and $I$ stands for the imaginary part) 


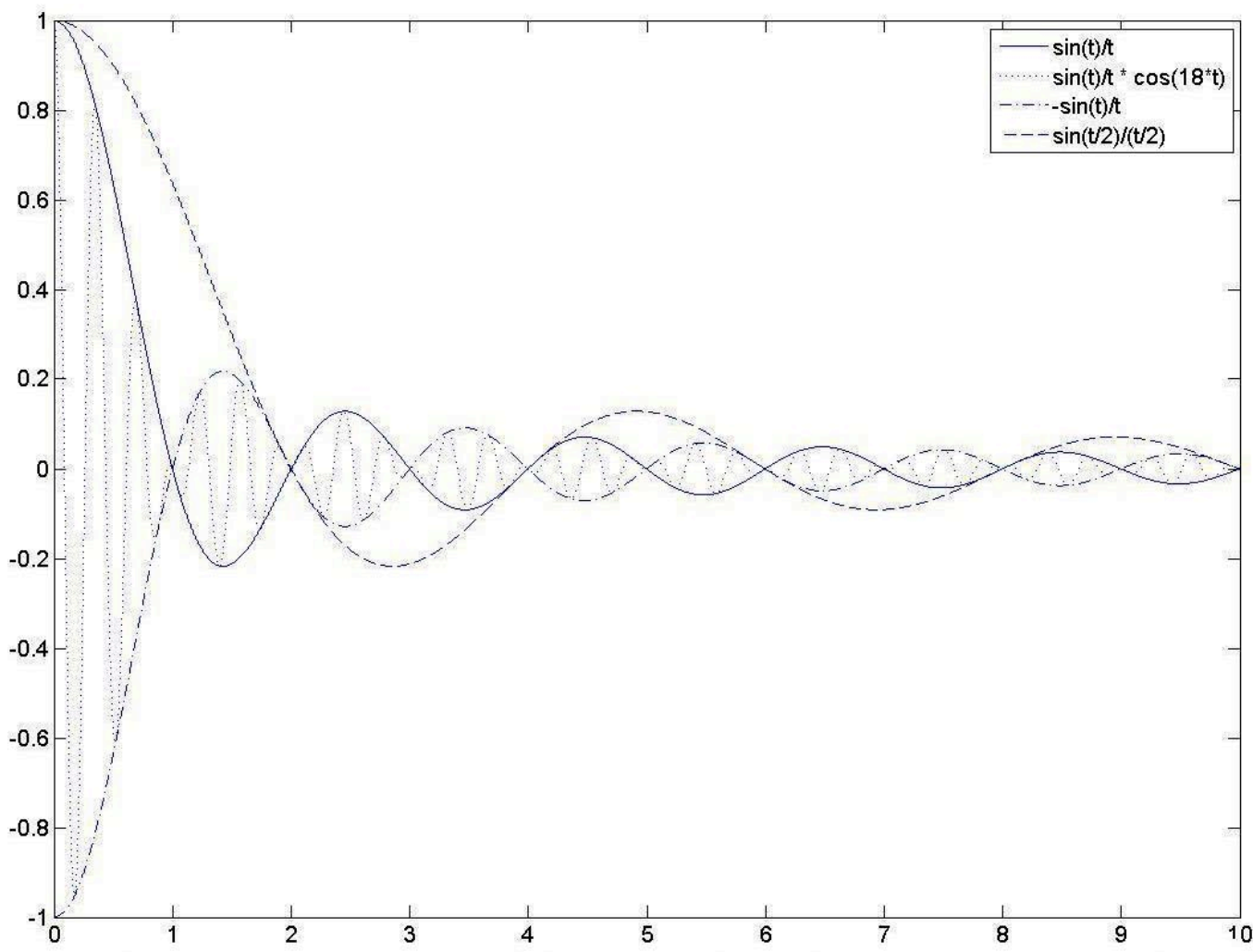

Fig. (2). Modulated signal (...) and its envelope (-), envelope of the signal (---).

$\tilde{f}(t)=\frac{e^{i \omega_{0} t}}{\sin \omega_{0}\left(\varphi_{1}-\varphi_{2}\right)}\left\{e^{i \omega_{0} \varphi_{1}} I\left[\widetilde{D}_{\omega_{0}}^{\varphi_{2}}[f](t)\right]-e^{i \omega_{0} \varphi_{2}} I\left[\widetilde{D}_{\omega_{0}}^{\varphi_{1}}[f](t)\right]\right\}$

provided that $\omega_{0}\left(\varphi_{1}-\varphi_{2}\right) \neq 0(\bmod 2 \pi)$.

Formula (10) provides the analytic signal $\tilde{f}$ of $f$ in function of the analytic signals $\widetilde{D}_{\omega_{0}}^{\varphi_{1}}[f], \widetilde{D}_{\omega_{0}}^{\varphi_{2}}[f]$ for two demodulations of $f$ with the same frequency downshifting $\omega_{0}$.

\section{REMARKS}

1) In radio communications, the amplitude modulation begins with a signal in baseband which is frequency upshifted in some frequency band for propagation. At the receiver the signal is frequency downshifted in baseband, using the frequency carrier of the modulation. In this situation the received signal is identical to the transmitted signal except for a time delay. Then envelopes at the transmitter and at the receiver are identical. But $g(t)$ (the signal in baseband) and $f(t)=2 g(t) \cos \omega_{0} t$ (the modulated signal) do not have the same envelope. For instance, with $\omega_{0}=18$ we have the following sequence

$$
\begin{aligned}
& g(t)=\int_{-1}^{1} e^{i \omega t} d \omega=\frac{2 \sin t}{t} \\
& \tilde{g}(t)=\frac{4 \sin (t / 2)}{t} e^{i t / 2}
\end{aligned}
$$

while we have

$$
\begin{aligned}
& f(t)=\frac{4 \sin t}{t} \cos 18 t \\
& \tilde{f}(t)=\int_{-1}^{1} 2 e^{i(\omega+18)} d \omega=\frac{4 \sin t}{t} e^{18 i t}
\end{aligned}
$$

The reason: in the computation of the analytic signal, only the positive part of the spectrum is taken into account. In Fig. (2) we show the signal in baseband, its envelope and the modulated version. Its envelope and the signal in baseband are proportional.

2) In optical communications, we may admit that the transmitter is monochromatic with some frequency $\omega_{1}>0$ $\left( \pm \omega_{1}\right.$ in the complex model). The propagation widens the beam spectrum so that the returned beam $f(t)$ has a continuous spectrum in some limited set $\left(-\omega_{1}-\delta,-\omega_{1}+\right.$ $\delta) \cup\left(\omega_{1}-\delta, \omega_{1}+\delta\right)$. As explained above, a demodulation of frequential amplitude $\omega_{1}$ will change the envelope. But the latter will be unchanged in demodulation with frequency shift $\omega_{0}>0$ such that $0<\omega_{0}<\omega_{1}-\delta$. Actually, we see that such a demodulation does not create aliasing.

3) Let a real function $\mu(t)$ such that ( $a>0)$ :

$e^{-i \mu(t)}=\int_{-a}^{a} \theta(\omega) e^{i \omega t} d \omega$.

The Fourier transform of $e^{-i \mu(t)}$ cancels outside of $(-a, a)$, If $\omega_{0}-a>0$ we have in the same time

$f(t)=\cos \left(\omega_{0} t-\mu(t)\right), H[f](t)=\sin \left(\omega_{0} t-\mu(t)\right)$

and then $\tilde{f}(t)=e^{i\left(\omega_{0} t-\mu(t)\right)}$.

Moreover, if the real function $r(t)$ is such that

$r(t)=\int_{-b}^{b} R(\omega) e^{i \omega t} d \omega, 0<b<\omega_{0}-a$

the Bedrosian's theorem [6] implies that the analytic signal of

$$
g(t)=r(t) \cos \left(\omega_{0} t-\mu(t)\right)
$$




$$
\tilde{g}(t)=r(t) e^{i\left(\omega_{0} t-\mu(t)\right)}
$$

Actually, most of the people accept implicitly that these restrictive hypotheses about the bandwidths of $r(t)$ and $e^{-i \mu(t)}$ with respect to $\omega_{0}$ are verified, and then they admit the last representation (12) . To assume a bounded spectrum for these quantities is a risky bet. Among reasons, they are non-linear functions of the studied function $f$. It is easy to give counter-examples, for instance defining $\mu(t)$ as a periodic rectangular function.

\section{CONCLUSION}

The concept of envelope is used in radio communications [5], laser propagation [1], radar backscatter [7]. The envelope is often measured looking at the analytic signal after demodulation which allows to work in baseband. In this paper, we show that the result is different of the signal envelope in its original frequency band except some cases (for instance when the Bedrosian's theorem can be used). We give a method to compute the true envelope (and the true phase) with two and not one demodulations. This paper is written in the functions framework taking into account a deterministic context, easier to handle than a random one. Properties are still true in the framework of stationary random processes. In classical amplitude modulationdemodulation, a signal in baseband $\mathrm{A}$ is sent in another frequency band (for instance multiplying by $\cos \omega_{0} t, \omega_{0}$ large enough). The result $\mathrm{B}$ is returned in baseband and lowpass filtered. The result $\mathrm{C}$ is equal to $\mathrm{A}$ (except for a multiplicative constant). Then the envelopes of $\mathrm{A}$ and $\mathrm{C}$ are proportional and the phases differ from $\omega_{0} t$ (except for an additive constant). Actually this property is untrue for $\mathrm{B}$ and $\mathrm{C}$ (or A and B). Consequently, the envelope and the phase of a laser beam (then in a high frequency band like B) can become very different after demodulation in baseband. This drawback is not taken into account in laser communication literature.

\section{CONFLICT OF INTEREST}

The authors confirm that this article content has no conflicts of interest.

\section{ACKNOWLEDGEMENTS}

Declared none.

\section{REFERENCES}

[1] Ridley KD. Measurements of laser phase fluctuations induced by atmospheric turbulence over $2 \mathrm{~km}$ and $17.5 \mathrm{~km}$ distances. Appl Opt 2011; 50(26): 5085-92.

[2] Sprenger B, Zhang J, Lu ZH, Wang LJ. Atmospheric transfer of optical and radio frequency clock signals. Opt Lett 2009; 34(7): 965-7.

[3] Djerroud K, Acef O, Clairon A, et al. Coherent optical link through the turbulent atmosphere. Opt Lett 2010; 35(9): 1479-81.

[4] Mandel L, Wolf E. Optical coherence and quantum optics. Cambridge: Cambridge University Press 2008.

[5] Papoulis A. Signal analysis. USA: Mac-Graw-Hill 1977.

[6] Schreier PJ, Scharf LL. Statistical signal processing of complexvalued data. Cambridge: Cambridge University Press 2010.

[7] Li X, Xu X. Numerical study on the phase and amplitude statistics of backscatter signals from time-evolving sea surfaces. IET Radar Sonar Nav 2011; 5(5): 551-60. 